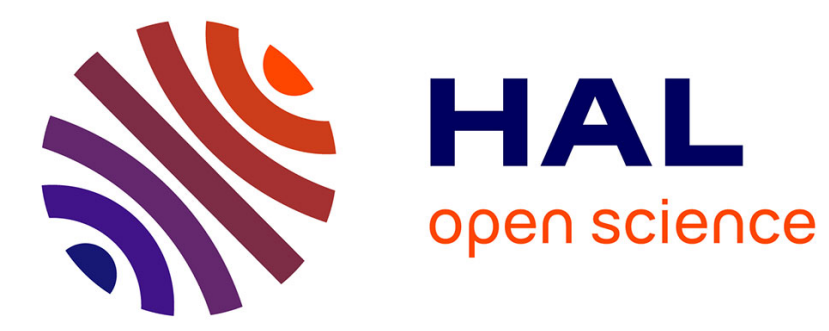

\title{
Structural relaxation and conductivity relaxation in glassy ionics
}

\author{
K. Ngai
}

\section{To cite this version:}

K. Ngai. Structural relaxation and conductivity relaxation in glassy ionics. Journal de Physique IV Proceedings, 1992, 02 (C2), pp.C2-61-C2-73. 10.1051/jp4:1992207 . jpa-00251278

\section{HAL Id: jpa-00251278 https://hal.science/jpa-00251278}

Submitted on 1 Jan 1992

HAL is a multi-disciplinary open access archive for the deposit and dissemination of scientific research documents, whether they are published or not. The documents may come from teaching and research institutions in France or abroad, or from public or private research centers.
L'archive ouverte pluridisciplinaire HAL, est destinée au dépôt et à la diffusion de documents scientifiques de niveau recherche, publiés ou non, émanant des établissements d'enseignement et de recherche français ou étrangers, des laboratoires publics ou privés. 


\title{
Structural relaxation and conductivity relaxation in glassy ionics
}

\author{
K. L. NGAI
}

Naval Research Laboratory, Washington, D. C. 20375, U.S.A.

The coupling model was proposed to describe relaxations in complex correlated systems of which ionic conducting glass is a special case. The model is capable of explaining both the structural and the conductivity relaxations of ionic conducting glasses. Recent results from Monte Carlo simulations of ionic motion in glasses are shown to be in total agreement with the predictions of the coupling model. Experimental data from nuclear spin relaxation in poor ionic conductors and quasielastic neutron scattering in fast ionic conductors provide also strong support for the validity of the coupling model. A correlation between the stretch exponent of conductivity relaxation with the decoupling index proposed by Angell is discussed in the light of the coupling model. 


\section{Introduction}

An ionically conducting glass provide two challenging problems in the field of the study of relaxation in complex systems. The glassy matrix leads us to the problem of understanding the structural relaxation of glass-forming liquids which is currently of great interest (1). The ions and their motions in either the glassy or the liquid state of the matrix provide the other problem of finding the mechanism of ionic diffusion and conductivity relaxation. We are fortunate that previous experimental studies over the last few decades of classical glassy ionic systems have bestowed us with rich phenomenologies (2), (3). Recent advances made in new glasses, particularly those which are fast ion conductors (1), have enriched the field immensely. In the last ten years we have witnessed constantly increasing theoretical efforts to understand the ionic conductivity relaxation. Included are the coupling model ( $4-12)$ based on general physical principles $(13-16)$, various theoretical models $(17-26)$ as well as Monte Carlo computer simulations $(27-29)$. While some of these theoretical efforts are consistent with each other in physical basis and the results, there remains the problem that several models cannot be reconciled with each other. Naturally, in physical sciences the only way to find out which theoretical approach is the correct one is to subject these different theories to stringent tests by comparing their predictions with experimental data and computer simulations data. In doing so, the proponents of a theory or model must not select only a certain set of experimental data, but to include all known facts. This is a difficult task because there are so many varieties of glassy ionic conductors and so many known facts about them have accumulated over the years. It is however absolutely unavoidable because failure to make a complete check of consistency of a theory with all known experimental data is tantamount to walking through a mine field without knowing where the mines are. Also the phenomenology of conductivity relaxation seen in many ionic glasses is similar to that seen in molten salts $0.4 \mathrm{Ca}\left(\mathrm{NO}_{3}\right)_{2}-0.6 \mathrm{KNO}_{3}(30)$, in electrolyte solutions such as $\mathrm{LiCl}$ water systems (31) and in $\mathrm{Na} \beta-\mathrm{Al}_{2} \mathrm{O}_{3}(3,4,12)$ which is not a glass. As pointed out by Moynihan (32), these similarities require that a viable theory or model be structure independent.

Many years ago $(13,33)$ since I proposed the coupling model to describe relaxations in complex correlation systems, (CCS) of which many ionic glasses are examples, I have paid a lot of attention to consistency of its predictions with established phenomenologies and experimental data, past and present $(2-12)$. In this work I shall make further comparisons of the predictions with recent Monte Carlo simulations $(27-29)$, NMR spin relaxation $(9,34-36)$ of poor ionic conductors and quasielastic neutron scattering $(37,38)$ experimental data of fast ionic conductors. All these facts have provided critical tests of the coupling model and should do the same for any other model. I may add that the coupling model has been applied to various relaxations in other complex correlated systems including structural relaxation in glass-forming liquids (39), viscoelasticity of polymers (8) and etc. with equal success. These feats should not be overlooked by workers even though their interest is focussed on one field such as ionically conducting glasses.

\section{The Coupling Model}

Diffusion of ions in glasses where the ions are interacting with each other is an example of a general class of problems what I called (40) relaxations in complex correlated systems (CCS). In any CCS the relaxation of the individual units (ions here) are correlated by their mutual interactions and hence the relaxations of these units are necessarily cooperative in nature. The problem is how to describe irreversible process (relaxation) in a system with many-body interactions. A rigorous analytic solution to this problem is extremely difficult and is unavailable at this time. Several attempts (13 - 16), collectively described as the coupling model, have been made to deduce the dynamics of these CCS from general basic physics of interacting many-body systems. For example in reference (15) we use the chaotic nature of the many-body interaction Hamiltonian and the associated fractal character of the phase space to derive the general result that the one-body relaxation rate $W_{0}$ after a characteristic time, $t_{c}=1 / \omega_{c}$ will be slowed down by many-body correlations to assume a power-law time dependence of $W(t)=W_{o}\left(\omega_{c} t\right)^{-n}$, where $o<n<1$ is the coupling parameter. The result being general and applicable for all CCS is extremely powerful. It is the physical law governing relaxations in CCS. The shortcoming of this approach is that $\mathrm{W}_{0}, \omega_{\mathrm{c}}$ and $\mathrm{n}$ are not calculated for any CCS so far, although in many applications $\mathrm{W}_{\mathrm{o}}$ is in principle known (8). Hence we have essentially two parameters $\omega_{c}$ and $n$. It is fortunate that $W(t)$ spawns (8) a number of predictions. On comparing the stretched-exponential relaxation function, $\phi(t)=\exp -\left(t / \tau^{*}\right)^{1-n}$ 
predicted from $\mathrm{W}(\mathrm{t})$ with the shape of the experimental relaxation function, $\mathrm{n}$ can be determined. With $\mathrm{n}$ determined, the other prediction $\tau^{*}=\left[(1-n) \omega_{c}^{n} \tau_{0}\right]^{1 /(1-n)}$ where $\tau_{0}=W_{0}^{-1}$, will generate several meaningful and stringent tests of the coupling model. The dependence of the "one-particle" (primitive) relaxation time $\tau_{\mathrm{o}}$ on various variables $V_{1}, V_{2}, \ldots$ will be modified for $\tau^{*}$ to $\tau^{*}\left(V_{1}, V_{2}, \ldots\right) \sim\left[\tau_{0}\left(V_{1}\right.\right.$, $\left.V_{2}, \ldots\right]^{1 /(1-n)}$. These predictions of the form of the scaling dependence on variables of $\tau^{*}$ based on the usually known dependence of $\tau_{0}$ on the same variables becomes parameterless once $n$ has been determined (8). To be fair, there is at present no model of ionically conducting glasses that contains no parameters. Given an ionic glass, say $\mathrm{xNa}_{2} \mathrm{O}-(1-\mathrm{x}) \mathrm{SiO}_{2}$ there is no theory that can calculate accurately the dc conductivity as a function of temperature and the conductivity relaxation as a function of time from first principles without introducing any parameters. The immense difficulty of the problem is because we are dealing with an irreversible process (relaxation) in a many-body interacting (correlated) system that is not ordered (complex). The above remarks apply also to "toy" models which replace the real ionic glass by mathematical constructs which by their very nature involve parametrizations of the input physics. Of course, a "toy" model would be useless if the input physics is incorrect. The purpose of my comments here is to point out that realistically, at the present time, none of the theories of the dynamics in ionic glasses is parameter free. Nevertheless a positive step forward has been made by a theory such as the coupling model which has many predictions. Use of one prediction and its comparison with that corresponding aspect of the experimental data determine the parameter $n$. Once $n$ is determined, many remaining predictions become parameterless and they have been tested rigorously. If one is willing to look into relaxations in other CCS beyond ionic glasses, then the parallel or similar successes the coupling model (8) has in these areas necessarily strengthen the support for the model.

If the rate slowing down result, $W(t)=W_{0}\left(\omega_{c} t\right)^{-n}$, of the coupling model ultimately had been established by basic physical principles on rigorous grounds, then it would become a physical law that anyone should feel comfortable to use. Currently we have started a theoretical effort in this direction (16) based on classical nonintegrable (chaotic) Hamiltonian and statistical mechanics of irreversible processes. Let me assume for the time being that it is indeed a physical law. Then I argue, in conformity with standard practice in other activities of statistical mechanics, there is no need to calculate the quantity $\mathrm{n}$ for any real relaxation process in a real material. It is perfectly respectable to measure $\mathrm{n}$ by an experiment which relates to one of several theoretical predictions in which $n$ appears, say the shape of the relaxation function, $\exp -\left(t / \tau^{*}\right)^{1-n}$. Analogies can be found in the many laws of statistical mechanics in which the temperature $T$ appears as a parameter. In applying these laws to a real physical system no one demands the temperature of the system to be calculated. But, instead, $T$ is measured by a separate experiment like the linear expansion of a liquid which is proportional to $T$, i.e. by a thermometer. After $T$ is determined by experiment, the other laws of statistical mechanics can be tested by experiments. Similarly applying the coupling model, after $\mathrm{n}$ is determined by a measurement of the time dependence or frequency dependence I can use this same $n$ to predict other nontrivial properties of relaxation in the CCS. Examples of these meaningful applications of the coupling model to explain various experimental data and to resolve anomalous properties have been given in several CCS including the glassy ionics (8). Of course the analogy between $\mathrm{T}$ and $\mathrm{n}$ used here should not be carried to far. Temperature is a much more fundamental physical quantity than the coupling parameter of the coupling model.

For the remainder of this paper we discuss mainly conductivity relaxation of ionic glasses. The problem of diffusion of ions with mutual interactions on a lattice, was formulated by Ngai and Liu (41), and Rendell et al. (41) as a continuous-time random walk (CTRW) process with waiting-time distribution, $\Psi(t)=-d \phi / d t$, where $d \phi / d t=-W_{0} \phi$ for $\omega_{c} t \leq 1$ and $d \phi / d t=-W_{o}\left(\omega_{c} t\right)^{-n} \phi$ for $\omega_{c} t>1$. The latter is the rate equation of the slowed down transition for $\omega_{\mathfrak{c}} t \geq 1$ from one site to another. The solutions to these rate equations are:

where

$$
\phi(t)=\exp \left[-\left(t / \tau^{*}\right)^{1-n}\right], \quad \omega_{c} t \geq 1
$$

and

$$
\tau^{*}=\left\{(1-n) \omega_{c}^{n} \tau_{0}\right\}^{1 /(1-n)}
$$

$$
\phi(t)=\exp -\left(t / \tau_{0}\right), \quad \omega_{c} t \leq 1
$$


we have calculated $\left\langle\mathrm{r}^{2}(\mathrm{t})\right\rangle$ analytically but asymptotically (41), and also numerically (41). There are three time regimes where $\left\langle\mathrm{r}^{2}(\mathrm{t})\right\rangle$ behaves differently as

$$
<\mathrm{r}^{2}(\mathrm{t})>=\left\{\begin{array}{l}
6 \mathrm{D}_{0} \mathrm{t} \quad \omega_{\mathrm{c}} \mathrm{t}<1 \\
6 \omega_{c}^{-\mathrm{n}} \mathrm{D}_{0} \mathrm{t}^{1-\mathrm{n}} \quad \omega_{\mathrm{c}}^{-1} \quad<\mathrm{t}<\tau^{*} \\
6 \mathrm{Dt} \quad \mathrm{t}>\tau^{*}
\end{array}\right.
$$

where

and

$$
\mathrm{D}_{\mathrm{o}} \approx \mathrm{c}_{\mathrm{d}}^{2} \tau_{\mathrm{o}}
$$

$$
\mathrm{D} \approx \mathrm{c}^{2} / \tau^{*}
$$

In Eqs. (7) and (8) the quantity $c_{0}$ is the jump distance. The frequency dependence $\sigma(\omega)$ can be calculated directly from $\left\langle\mathrm{r}^{2}(\mathrm{t})\right\rangle$ by

which gives

$$
\sigma(\omega) \sim \omega^{2} \int_{0}^{\infty} d t \exp (i \omega t)<r^{2}(t)>
$$

$$
\sigma(\omega) \sim \begin{cases}\text { Const } / \tau_{\mathrm{o}} & \text { if } \omega>\omega_{\mathrm{c}} \\ \mathrm{A} \omega^{\mathrm{n}} / \tau_{\mathrm{o}} & \text { if } 1 / / \tau^{*}<\omega<\omega_{\mathrm{c}} \\ \text { Const } / \tau^{*} & \text { if } \omega<\left(\tau^{*}\right)^{-1}\end{cases}
$$

Alternatively, with the site-to-site transfer correlation function given by the stretch exponential Eq. (1), we can calculate electrical relaxation by the electrical modulus, $\mathbf{M}^{*}(\omega)$, formalism $(42,43,6)$

$$
M^{*}(\omega) \equiv 1 / \epsilon^{*}(\omega)=M_{s}\left[1-\int_{0}^{\infty} \mathrm{dt} \exp (-\mathrm{i} \omega \mathrm{t})(-\mathrm{d} \phi / \mathrm{dt})\right]
$$

where $M_{S}$ is the reciprocal of the limiting high frequency dielectric constant $\epsilon_{s} . M^{\prime \prime}(\omega)$, the imaginary part of $M^{*}(\omega)$, exhibits a skew asymmetric peak with peak frequency

$$
\omega_{\mathrm{p}} \sim 1 / \tau^{*}
$$

From $\mathrm{M}^{*}(\omega)$ we can calculate $(7,6,12), \in^{*}(\omega)$ and $\sigma(\omega)$ by Maxwell's relations. The ac conductivity calculated this way has exactly the dependences as given by Eqs. (10) - (12). The dc conductivity is given by

$$
\sigma_{\mathrm{dc}} \sim\left(\epsilon_{\alpha} \mathrm{M}_{\mathrm{S}}\right) / \tau^{*} \sim\left(\epsilon \mathcal{\alpha} \mathrm{M}_{\mathrm{S}}\right) \omega_{\mathrm{p}}
$$

\section{Monte Carlo Simulations}

The mean square displacement given by Eqs. (4) - (8) for interacting ionic systems from the coupling model are verified by Monte Carlo simulations by Maass, Petersen, Bunde, Dieterich and Roman [27] for Coulombically interacting ions in structurally disordered lattices in which a fraction of sites are inaccessible to the mobile ions. They found, in the presence of both disorder and interaction, $\left.<\mathrm{r}^{2}(\mathrm{t})\right\rangle$ has exactly the time-dependence as given by Eqs. (4) - (6). In an intermediate time regime $<\mathrm{r}^{2}(\mathrm{t})>\sim \mathrm{t}^{1-\mathrm{n}}, \mathrm{t}_{1}<\mathrm{t}<\mathrm{t}_{2}$ where $\mathrm{o}<\mathrm{n}<1$. The crossover time, $\mathrm{t}_{1}$, from Monte Carlo simulation, to be identified with $\omega_{r}^{-1}$ of the coupling model, is independent of temperature. This result verifies a property of $\omega_{c}$ as proposed in the coupling model. Continuity conditions between the three regimes of $\left\langle\mathrm{r}^{2}(\mathrm{t})\right\rangle$ given by Eqs. (4) - (6) at the boundaries $t_{1} \cong \omega_{c}^{-1}$ and $t_{2} \cong \tau *$ can be written down. These two equations together with the expressions for $D_{0}$ and $D$ from Eqs. (7) and (8) respectively lead to the relation between $\tau^{*}$ and $\tau_{\mathrm{o}}$ given by Eq. (2). Hence the Monte Carlo simulation has also verified this important relation which has been used repeatedly in the past to explain experimental data and anomalous 
properties $(7,8)$. Here, via Eqs. (15) and (2) we can see that it explains the anomalous isotope mass dependence of $\sigma_{\mathrm{dc}}$ observed in $\mathrm{Li}_{2} \mathrm{O} \cdot 3 \mathrm{~B}_{2} \mathrm{O}_{3}$ glasses (7). Since $\tau_{\mathrm{o}}$ has the normal isotope mass dependence of $\mathrm{m}^{1 / 2}$, from Eq.(2)it is clear that $\left(\tau^{*}\right)^{-1}$ as well as $\sigma_{\mathrm{dc}}$ will have the anomalous isotope mass

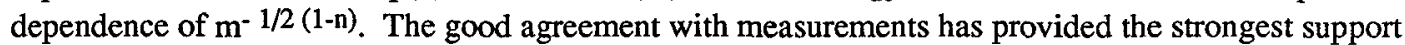
for the coupling model $(7,44)$. Monte Carlo simulations (27) found also that on increasing the interaction strength, the exponent $k^{\prime} \equiv 1-n$ in Eq. (5) decreases and the crossover time $\tau^{*}$ increases. This trend in $\mathrm{k}^{\prime}$ is expected also from the coupling model and the increase of $\tau^{*}$ is consistent with the relation (2) between $\tau^{*}, \tau_{0}$ and $n$. These properties are absent in single-particle (noninteracting) transport in infinite percolation clusters which are fractals although $\left\langle\mathrm{r}^{2}(\mathrm{t})\right\rangle$ has also a $\mathrm{t}^{\mathrm{k}}$ dependence (27-29).

Maass et al. (27) have given additional insights into the dynamics of ionic motion in the intermediate time regime where $\left\langle\mathrm{r}^{2}(\mathrm{t})\right\rangle \sim \mathrm{t}^{1-\mathrm{n}}$. They have determined the mean square displacement as a function of the number of performed hops, $N$. They found a striking alternation of $\left\langle r^{2}(N)\right\rangle$ for even and odd $\mathrm{N}$ which is the consequence of strong backward correlations in the tracer motion. Most particles, which have performed an odd number of jumps are out of equilibrium and have a large tendency to jump back to the site, which they have previously occupied. The time dependence of $\left\langle\mathrm{r}^{2}(\mathrm{t})\right\rangle$ is dominated by $\left\langle\mathrm{r}^{2}(\mathrm{~N})\right\rangle$ for even $\mathrm{N}$ and corresponding to equation (5) they found that

$$
<\mathrm{r}^{2}(2 \mathrm{~N})>\sim(2 \mathrm{~N})^{1-\mathrm{n}}, \quad \mathrm{N}_{1}<\mathrm{N}<\mathrm{N}_{2}
$$

where $N_{1}=W_{0} t_{1}, N_{2}=W_{0} t_{2}$ and $W_{0}$ is the mean jump rate of a particle. Equation (16) shows explicitly that the power law behavior of $\left\langle\mathrm{r}^{2}(\mathrm{t})\right\rangle$ or $\sigma(\omega)$ cannot be explained by a singular waiting time distribution. In this case $\left\langle\mathrm{r}^{2}(\mathrm{~N})\right\rangle$ would obey Fick's law, $\left\langle\mathrm{r}^{2}(\mathrm{~N})\right\rangle \sim \mathrm{N}$, and the power law in

$\left\langle\mathrm{r}^{2}(\mathrm{t})\right\rangle$ follows from the distribution of waiting times between consecutive jumps of the tracer particle. This result demonstrates the intricate behavior of relaxation in complex correlated systems. The relaxations of units are heterogeneous yet they cannot be treated by a distribution of relaxation or waiting times. Similar remarks apply to other complex correlated systems including the entangled polymer chains and the glass-forming viscous liquids (45). The results of computer simulations (46), (47) for mean square displacement of the center of mass have the same form as Eq. (4) - (8) for ions in conformity with the coupling model (14). The many-particle problem of ion transport in solid or glassy electrolytes when ion-ion correlations are important is a highly nonlinear one that cannot be solved rigorously and analytically. We have mentioned the coupling model introduced more than a decade ago as a compromise to treat this problem as well as other complex correlated systems including structural relaxation of glass-forming liquids and viscoelasticity of polymers. The Monte Carlo simulations of Maass et al. have added valuable insights into this problem. It is worthwhile to mention another model in the same vein proposed by Funke. To treat this complicated problem approximately, Funke $(17,18)$ has developed the semi-microscopic jump-relaxation model, which bears some similarity to the DebyeFalkenhagen theory. In the model, rate equations are written down that describe the correlated forwardbackward motion of a single ion in the effective potential of the surrounding ions. The jump-relaxation model yields much more dramatic dispersion in the ac-conductivity than the Debye-Falkenhagen theory. Even though the model is simple and involves some assumptions and several approximations, it could be applied successfully to describe several aspects of solid ionic conductors and obtain, for example, the form of the ac-conductivity, the spin-lattice relaxation time and neutron scattering spectra. His results summarized in Ref. 18 are almost identical to mine, which as discussed here were published much earlier ( $3-15,33,41,48)$. Unfortunately these earlier results which Funke has recaptured in his model were not cited in Ref. 18.

\section{NMR Spin Relaxation in Alkali Oxide Glasses}

For NMR spin relaxation, we should distinguish the ionic conductors with high conductivity (superionic) from those with low conductivity. The superionic conductors include the $0.5 \mathrm{AgPO}_{3}-0.5 \mathrm{Ag}$ I, $0.5 \mathrm{Ag}_{2} \mathrm{~S}-0.5 \mathrm{GeS}_{2}, \mathrm{Na} \beta-\mathrm{Al}_{2} \mathrm{O}_{3}, 0.5 \mathrm{Li}_{2} \mathrm{~S}-0.5 \mathrm{SiS}_{2}$ etc. The low conductivity systems include the alkali oxide glasses such as the silicates, borates, and germanates, and especially in glasses with lower alkali content (e.g., $\mathrm{Li}_{2} \mathrm{O}-3 \mathrm{~B}_{2} \mathrm{O}_{3}$ ). In superionic conductors, the motion of the ions is fast. Nuclear spins are relaxed by the motion of these fast ions and the nuclear maximum of the spin relaxation rate, $\mathrm{T}_{1}{ }^{-1}$, can be observed below the glass transition temperature. The frequency dependence of the 
maximum $T_{1}^{-1}$, as well as the magnitude of $T_{1}^{-1}$ at a fixed temperature below the temperature of $T_{1}^{-1}$ maximum is superlinear, having the form of $\omega_{\mathrm{L}}{ }^{-(2-n)}$ if the fractional exponential exp- $(\mathrm{t} / \tau)^{1-n}$ is used for the correlation function (48), (33), (4), (49-51). On the other hand, for low conductivity oxide glasses the motion of the ions being slow, the $\mathrm{T}_{1}{ }^{-1}$ maximum is usually not observed within the temperature range bounded above by the glass transition temperature, $T_{g}$. Throughout the temperature range (typically from $2 \mathrm{~K}$ to $700 \mathrm{~K}$ for the glasses we studied) the frequency dependence is sublinear, i.e., $\mathrm{T}_{1}{ }^{-1}$ $\propto \omega_{\mathrm{L}}^{-\alpha}$ where $0<\alpha<1$. In the low temperature region between $2 \mathrm{~K}$ to a couple of hundreds of Kelvin, the temperature dependence is a power law, $T^{\gamma}$ where $\gamma \sim 1.0$. The combined $\omega_{\mathrm{L}}$ and $T$ dependences of $(34-36,9)$ the spin relaxation rate given by

$$
\mathrm{T}_{1}^{-1} \propto \omega_{\mathrm{L}}^{-\alpha} \mathrm{T}^{\gamma} \quad 0<\alpha<1, \gamma \approx 1.0
$$

is typical of glasses (52 - 54)and has been observed in alkali oxide glasses as well as in oxide glasses without any alkali ion. The mechanism currently accepted is spin relaxation by a "Raman" process involving thermally activated transitions of two two-level-systems, or more exactly, two asymmetric double well potentials (ADWP) $(34-36)$. The distribution of potential energy barriers together with the nature of the Raman process $(34,54)$ leads to the mild power law dependence of $T_{1}^{-1}$ on $T$. The mechanism also leads to an almost linear frequency dependence similar to that observed in experiment. As temperature is raised above a few hundred degrees Kelvin, the sublinear frequency dependence persists, but the temperature dependence changes and becomes Arrhenius exp- $\left(\mathrm{E}_{a} / \mathrm{kT}\right)$ with a welldefined activation energy, $E_{2}$. These frequency and temperature dependences are confirmed by Heitjans and coworkers using $\beta$-NMR technique (35) which considerably extends the frequency range in which the sublinear dependence holds. The sublinear frequency dependence of $T_{1}^{-1}$ being the characteristic of spin relaxation by thermal activated Raman processes of ADWP's, it is natural to continue to interpret the data by such a mechanism in the Arrhenius regime where the sublinear frequency dependence continues to hold. The Arrhenius temperature dependence is explained by the addition of a local hopping of an alkali ion over the real energy barrier of height $\mathrm{E}_{\mathrm{a}}$ to the ADWP Raman processes and the latter relaxes the spin. In other words, at lower temperatures, spin relaxation is by ADWP Raman process without the transfer of ion from one site to another. At higher temperatures, ADWP Raman process is accompanied by an ion hop over the real energy barrier, $E_{\mathrm{a}}$, but the former remains to be the dominant spin relaxation mechanism. Thermal activated hopping of ion with a constant activation energy, $\mathrm{E}_{\mathrm{a}}$, gives rise to the Arrhenius temperature dependence exp- $\left(\mathrm{E}_{\mathrm{a}} / \mathrm{kT}\right)$ and the ADWP Raman process is again responsible for the sublinear frequency dependence and the mild $T^{\gamma}$ dependence to $T_{1}{ }^{-1}$. The combined frequency and temperature dependences of the spin relaxation rate are given approximately by

$$
\mathrm{T}_{1}^{-1} \propto \omega_{\mathrm{L}}^{-\alpha} \exp -\left(\mathrm{E}_{\mathrm{a}} / \mathrm{kT}\right), \quad 0<\alpha<1
$$

In this interpretation of the $T_{1}{ }^{-1}$ data we are led to identify the observed activation energy, $E_{a}$, as the real one particle energy barrier of ionic motion. This identification has great significance because $\mathrm{E}_{\mathrm{a}}$ is appreciably smaller than the activation energy for dc conductivity, $\mathrm{E}_{\sigma}$, and one could have identified the latter as the real energy barrier. Indeed most theories and models of ionic conduction identify $\mathrm{E}_{\sigma}$ and not $E_{\mathrm{a}}$ to be the real one-particle energy barrier. Only the coupling model has advocated the opposite. Of course results of Monte Carlo simulation support this coupling model interpretation because of the detailed agreements as discussed above. Therefore experimental data that can distinguish unequivocally which activation energy is the real one will be of great interest. The aforementioned data of spin-lattice relaxation rate, $T_{1}^{-1}$, in lower conductivity oxide glasses indicates that $E_{a}$ is the real one-particle energy barrier in agreement with the coupling model. In addition, comparison between $E_{a}$ and $E_{\sigma}$ obtained from experimental data have verified the predicted relation (9), (55)

$$
\mathrm{E}_{\mathrm{a}}=(1-\mathrm{n}) \mathrm{E}_{\sigma}
$$

between them. Here 1- $\mathrm{n}$ is the fractional exponent of the conductivity relaxation function in the electric modulus representation of dielectric response data.

This volume of experimental data obtained mainly in alkali oxide glasses (silicates, borates and germanates) remains to date the best method to provide the evidence to support $\mathrm{E}_{\mathrm{a}}$ in $\mathrm{Eq}$. (19) as the true one particle energy barrier for poor ionic conductors. However, the method is inapplicable to fast ion conductors. For fast ion conductors, diffusion of ions is sufficiently fast to dominate spin relaxation. Spin relaxation by ADWP's with or without the assistance of an ion hop is comparatively not as 
effective. These contributions are overwhelmed, except down at very low temperatures by ion diffusion. As a result, the contribution to $\mathrm{T}_{1}{ }^{-1}$ with a sublinear frequency dependence and an Arrhenius $\mathrm{T}$ dependence $\exp \left(\mathrm{E}_{2} / \mathrm{kT}\right)$ disappears. In its stead, $\mathrm{a} \mathrm{T}_{1}{ }^{-1}$ with a superlinear frequency dependence and $\exp \left(\mathrm{E}_{\mathrm{a}} / \mathrm{kT}\right)$ temperature dependence is measured throughout the temperature region below the temperature $\mathrm{T}_{\max }$ at which the $\mathrm{T}_{1}^{-1}$ maximum is observed. Such a superlinear frequency dependence comes from spin relaxation by diffusion of ions with the fractional exponential, Eq. (1), as the correlation function. In fact, this mechanism has (33), (4), (3), (48-51)

$$
\mathrm{T}_{1}{ }^{-1} \propto \omega_{\mathrm{L}}{ }^{2-\mathrm{n}} \exp -\left(\mathrm{E}_{\mathrm{a}} / \mathrm{kT}\right)
$$

on the low temperature side and a frequency independent

$$
\mathrm{T}_{1}{ }^{-1} \propto \exp -\left(\mathrm{E}_{\mathrm{a}} /(1-\mathrm{n}) \mathrm{kT}\right)=\exp -\left(\mathrm{E}_{\mathrm{a}}^{*} / \mathrm{kT}\right)
$$

on the high temperature side of the $T_{1}^{-1}$ maximum. Further the larger activation energy $E_{a}^{*} \equiv E a /(1-n)$ in Eq. (21) is identical to the dc conduction activation energy, $E_{\sigma}$. Hence there are two activation energies, $E_{a}$ and $E_{\sigma}$, from the NMR $T_{1}{ }^{-1}$ data of fast ionic conductors. From this data alone, there is no way to tell which one corresponds to the real energy barrier. Fortunately there are alternative experimental methods that can distinguish the real energy barrier for fast ionic conductors. Due to fast diffusion and short correlation time, the ion motion can be monitored by Brillouin scattering (BS) and by quasielastic neutron scattering (QENS) at higher temperatures but still below the glass transition temperature, $\mathrm{T}_{\mathrm{g}}$. Some experimental data of superionic conductors are available at this time. The implications of these results are the subjects of discussion in the following section. We shall show the QENS data provide additional evidence that the real energy barrier is not $\mathrm{E}_{\sigma}$ but a smaller $\mathrm{E}_{\mathrm{a}}$ which can also be identified with the product (1-n) $\mathrm{E}_{\sigma}$ as suggested by the coupling model.

\section{Quasi-elastic Neutron Scattering in $\mathrm{AgI}-\mathrm{AgPO}_{3}$ and $\mathrm{Ag}_{2} \mathrm{~S}-\mathrm{GeS}_{2}$}

The glassy systems $\left(\mathrm{Ag}_{2} \mathrm{~S}\right)_{\mathrm{x}}-\left(\mathrm{GeS}_{2}\right)_{1-\mathrm{x}}$ are superionics with high ionic conductivities. For composition with $\mathrm{x}=0.5$, the system exhibits negligible electronic conduction (56). The high conductivities are achievable without the incorporation of AgI, as in the other superionic conducting systems, $\mathrm{x}\left(\mathrm{AgPO}_{3}\right)-(1-\mathrm{x}) \mathrm{AgI}$, which (38) can complicate interpretation due to uncertainty in whether small clusters of AgI with tetrahedral coordination are dispersed in the $\mathrm{AgPO}_{3}$ based glassy structure. From small-angle neutron scattering studies the $\left(\mathrm{Ag}_{2} \mathrm{~S}\right)_{0.5}-\left(\mathrm{GeS}_{2}\right)_{0.5}$ glass appears to be more homogeneous than many other glasses with comparable ionic conductivities. These advantages render interpretation of the quasi-elastic neutron scattering experimental data relatively simple and clear for the superionic to be discussed.

Pradel and Ribes (56) have made measurements of the frequency dependent conductivity $\operatorname{Re}^{*}(\omega)$ from $10 \mathrm{~Hz}$ to $13 \mathrm{MHz}$ over a temperature range of $130 \mathrm{~K}$ to $500 \mathrm{~K}$. Arrhenius plot of $\operatorname{Re} \sigma^{*}(\omega)$ at constant $\omega$ reveals the two regimes given by Eqs. (11) and (12) and their crossover. With $\tau^{*}$ given by $\tau^{*}=\tau_{\infty}^{*} \exp \left(E_{a}^{*} / k T\right)$, at sufficiently low temperatures, the condition $\omega \tau^{*} \gg>1$ is satisfied and Eq. (11) together with $W_{0}=v_{\infty} \exp \left(-E_{a} / k T\right)$ predicts

$$
\operatorname{Re} \sigma^{*}(\omega) \propto \omega^{n} \exp \left[-(1-n) E_{d c} / k T\right]
$$

On the other hand, at high temperatures the condition $\omega \tau^{*}<<1$ holds and Eq. (12) predicts

$$
\operatorname{Re}^{*}(\omega)=\sigma_{\mathrm{dc}} \propto \exp \left(-\mathrm{E}_{\mathrm{dc}} / \mathrm{kT}\right)
$$

From the ac conductivity data of Pradel and Ribes, the dc conductivity has an Arrhenius dependence from which the activation energy

$$
\mathrm{E}_{\mathrm{dc}}=0.34 \mathrm{eV}
$$

has been deduced. At high frequencies and low temperatures, the experimental data offers two ways to deduce $\mathrm{n}$ or $\beta \equiv 1-\mathrm{n}$. The first way is via the measured temperature dependence of the ac conductivity $\operatorname{Re} \sigma^{*}(\omega)$ which is also Arrhenius but with a reduced activation energy, $E_{\mathrm{ac}}=0.15 \mathrm{eV}$. This is in agreement with what is predicted directly from Eqs. (11) and (22), the latter identifies

$$
(1-n) E_{d c}=E_{a c}=0.15 \mathrm{eV}
$$

Substituting the value of $E_{\mathrm{dc}}$ from Eq. (24) into (25), we obtain the value

$$
1-n=0.44
$$


The second method to obtain $\mathrm{n}$ is from the $\omega^{\mathrm{n}}$ dependence of $\operatorname{Re} \sigma^{*}(\omega)$ in Eq. (21). At a constant temperature corresponding to $10^{3} / \mathrm{T}=7.0, \operatorname{Re} \sigma^{*}(\omega)$ at four frequencies $10^{4}, 10^{5}, 10^{6}$, and $10^{7} \mathrm{~Hz}$ is in conformity with a $\omega^{\text {n }}$ dependence with

$$
n \simeq 0.56
$$

Thus, both the frequency and temperature dependences of the ac conductivity data can be represented in the form of Eq. (22) with the same value of $n$. The ac conductivity data of Pradel and Ribes are in accord with the description of conductivity relaxation based on the stretched exponential correlation function and the coupling model. Indeed there are two activation energies, $\mathrm{E}_{\mathrm{dc}}$ and $\mathrm{E}_{\mathrm{ac}}$, related by $E_{a c}=(1-n) E_{d c}$. However, this data alone does not tell us which one corresponds to the real one-particle energy barrier.

Superionic conductors with large ionic diffusion coefficients can be studied by quasi-elastic neutron scattering (QENS) because ionic diffusion induces quasi-elastic broadening in the neutron timeof-flight spectrum $(37,38)$. The same glass $\mathrm{Ag}_{2} \mathrm{~S}-\mathrm{GeS}_{2}$ studied by conductivity relaxation and discussed in the last section has high ionic mobility. QENS measurements were performed in this glass by Owens et al. This system was chosen for QENS studies because of its negligible electronic conduction, its high conductivity without the necessity of addition of AgI which may complicate the structure, and its greater homogeneity than many other glasses with comparable ionic conductivities. Time of flight spectra were measured at constant temperature. From these measurements, the quasielastic $(\mathrm{Ag}-\mathrm{Ag})$ structure factor $\mathrm{S}(\mathrm{Q})$ was obtained at scattering vector $\mathrm{Q}$. A plot of the product $\mathrm{S}(\mathrm{Q}) \Delta \mathrm{E}$ versus $\mathrm{Q}^{2}$, where $\Delta \mathrm{E}$ is the half width at half maximum, is a straight line whose slope yields the diffusion coefficient, $D$. The temperature dependence of $D$ was deduced from these isothermal plots. An average activation energy for $\mathrm{D}$ of $0.15 \mathrm{eV}$ was obtained. The activation energy for the $\mathrm{Ag}^{+}$diffusion coefficient obtained from the QENS data is about one half that found for the dc conductivity, $E_{\mathrm{dc}}$, given by Eq. (24), and is comparable to $\mathrm{E}_{\mathrm{ac}}$ for the ac conductivity given by Eq. (25).

The observation of an appreciable difference in the activation energy of the $\mathrm{Ag}^{+}$ion diffusion coefficient $\mathrm{D}$ obtained by $\mathrm{QENS}$ measurements from that found for the dc conductivity poses difficulty for models of conductivity that relate $\mathrm{E}_{\mathrm{dc}}$ to the real energy barrier (19-22). Without any modification or additional assumption, these models would expect to find the activation energy of $D$ to be exactly $E_{d c}$. To resolve this discrepancy in activation energies, Owens et al. offer the following explanation. The characteristic time scale in the QENS measurements is between $10^{-10}$ to $10^{-12} \mathrm{~s}$. The QENS measurements are monitoring diffusive motion at very high frequencies (say with an average frequency of $10^{11} \mathrm{~Hz}$ ). They found the diffusion coefficient obtained from the QENS measurements is about 40 times that estimated from the dc conductivity at the same temperature of $25^{\circ} \mathrm{C}$. They also extrapolated the ac conductivity to $10^{11} \mathrm{~Hz}$ and found the ac conductivity at this frequency is approximately 70 times larger than the dc value at $25^{\circ} \mathrm{C}$. From these reasonings Owens et al. (35) asserted that QENS measurements are equivalent to ac conductivity at high frequencies of the order of $10^{11} \mathrm{~Hz}$, both are monitoring motion of $\mathrm{Ag}^{+}$ions. The activation energy for the diffusive coefficient deduced from the QENS results is therefore comparable with the activation energy deduced from the temperature dependence of the ac conductivity. In order for this explanation to be complete, one needs to address the central question of why the ac conductivity has a considerably smaller activation energy, $E_{a c}$, than that for dc conductivity, $\mathrm{E}_{\mathrm{dc}}$. To answer this question, a model of the motions of ions is needed. Most proposed models, including that discussed by Elliott and Owens (20-24), are committed to the identification of $E_{\mathrm{dc}}$ but not $E_{\mathrm{ac}}$ to be the real energy barrier or activation energy of ion motion. This identification of $E_{d c}$ to be the real energy barrier contradicts the QENS data. The contradiction will become evident if we reexamine the method of analyzing QENS data. The time-of-flight spectra after standard corrections were transformed into the scattering law, $S(Q, \omega)$, as a function of energy transfer at constant scattering angle, $Q$. The energy half-width, $\Delta E$, of the quasielastic peak is determined by fitting $\mathrm{S}(\mathrm{Q}, \omega)$ by the expression

$$
S(Q, \omega)=A\left\{\left[A_{o}(Q) \delta(\omega)+\left[1-A_{o}(Q)\right] \pi^{-1}\left[\Delta E /\left[(\Delta E)^{2}+\omega^{2}\right]\right] \otimes R(\omega)\right\}+B\right.
$$

which takes into consideration the inelastic background, $B$, the purely elastic contribution, $A_{0}(Q)$, and the energy resolution function, $\mathrm{R}(\omega)$. $\mathrm{A}$ is an amplitude and the symbol $\otimes$ denotes a convolution (38). From the fit to the data, the parameters $\mathrm{A}, \mathrm{B}$, and $\triangle \mathrm{E}$ are determined. The quasielastic structure factor, $\mathrm{S}(\mathrm{Q})$, of the mobile $\mathrm{Ag}^{+}$ion is obtained by summing over the QENS line. The product $\mathrm{S}(\mathrm{Q}) \triangle \mathrm{E}$ is plotted versus $\mathrm{Q}^{2}$. Such a plot yields a straight line the slope of which is the time independent diffusion 
coefficient, $\mathrm{D}$. This procedure of determining $\mathrm{D}$, particularly the Lorentzian form of the quasielastic part of $S(Q, \omega)$ given by Eq. (28), is consistent with an exponential correlation function exp(-DQ $\left.{ }^{2} t\right)$ and $\left\langle\mathrm{r}^{2}\right\rangle=6 \mathrm{Dt}$. These results from QENS experiment leave no alternative but to interpret $\mathrm{D}$ so determined as the diffusion coefficient that relates to the dc conductivity at the same temperature by the familiar relation between dc conductivity, $\sigma_{\mathrm{dc}}$, and diffusion constant $\mathrm{D}$ :

$$
\mathrm{D}=\sigma \mathrm{Ne}^{2} / \mathrm{kT}
$$

It is also clear by comparing the corresponding expressions of $\sigma(\omega)$ and $\left\langle r^{2}(t)>\right.$ in the same time regime given by Eqs. (11) and (5) respectively that ac conductivity invoked in the explanation by Owens et al. should correspond to an anomalous diffusion with $\left\langle\mathrm{r}^{2}(\mathrm{t})\right\rangle \propto \mathrm{t}^{1-\mathrm{n}}$ which cannot be reconciled with the Lorentzian line shape a $\mathrm{Q}^{2}$ dependence for the product $\mathrm{S}(\mathrm{Q}) \Delta \mathrm{E}$ and $\left\langle\mathrm{r}^{2}(\mathrm{t})\right\rangle=6 \mathrm{Dt}$ as found experimentally $(37,38)$. These contradictions invalidate the explanation offered by Owens et al $(37)$.

At high temperatures when $\tau^{*}$ becomes short, say less than $10^{-10} \mathrm{~s}$, there is experimental evidence from fast-ion-conducting glasses including $(\mathrm{AgI})_{\mathrm{x}}-\left(\mathrm{Ag}_{2} \mathrm{O}-2 \mathrm{~B}_{2} \mathrm{O}_{3}\right)_{1-\mathrm{x}}$ and $(\mathrm{AgI})_{\mathrm{X}}-(\mathrm{AgPO})_{1-\mathrm{x}}$ that the relaxation spectrum narrows dramatically towards that of exponential relaxation as $\tau^{*}$ decreases (58-60). In the description of the relaxation function by the Kohlrausch form, the exponent (1-n) increases towards unity as $\tau^{*}$ decreases with temperature. As a result of this and Eq. (18) from the coupling model, the activation energy, $E_{\mathrm{a}}^{*}$, of $\tau^{*}$ will decrease toward the smaller real one particle activation energy, $\mathrm{E}_{\mathrm{a}}$, of $\tau_{\mathrm{o}}$ as $\tau^{*}$ decreases. We expect this to be true also for $\mathrm{Ag}_{2} \mathrm{~S}-\mathrm{GeS}_{2}$ glasses. This explanation can be checked by comparing the product $(1-n) E_{a}^{*}$, where $(1-n)$ and $E_{a}^{*}$ are taken from conductivity relaxation data at lower temperature, with $E_{n}$ obtained from neutron scattering. The value of (1-n) can be obtained from the fits of the experimental ac conductivity data of $\sigma(\omega)=\sigma(0)+A \omega^{\mathrm{s}}$, and identifying $\mathrm{s}$ with $\mathrm{n}$. For $\mathrm{Ag}_{2} \mathrm{~S}-\mathrm{GeS}_{2}$, Pradel et al. $(37,56)$ have determined $\mathrm{E}_{\mathrm{a}}^{*}$ to be $0.34 \mathrm{eV}$ and $\mathrm{s}$, varying slightly with temperature, to have a mean value of 0.55 . The product $(1-n) E_{a}^{*}$ then has the value of $0.153 \mathrm{eV}$ which is remarkably close to $0.15 \mathrm{eV}$, the value of $\mathrm{E}_{\mathrm{n}}$. Similarly for $0.5 \mathrm{AgPO}_{3}-0.5 \mathrm{AgI}$, Dianoux et al. (36) have given $E_{\sigma}^{*}=21 \mathrm{~kJ} / \mathrm{mol}$. and $E_{n}=8.7 \mathrm{~kJ} / \mathrm{mol}$. Conductivity relaxation data of $\mathrm{Liu}$ and Angell (49) gives us an estimate of 0.56 for $n$. Hence the product $(1-n) \mathrm{E}_{\mathrm{a}}^{*}$ evaluates to 9.24 $\mathrm{kJ} / \mathrm{mol}$, which again is close to the activation energy $E_{n}$ of the diffusion constant $D$ determined by neutron scattering experiment. Thus we may conclude that the coupling model has provided a satisfactory explanation of the quasi-elastic neutron scattering. Most importantly the neutron data provides strong evidence that the one-particle energy barrier is indeed $E_{a} \equiv(1-n) E_{a}^{*}$ and not $E_{a}^{*}$.

\section{Decoupling of Conductivity Relaxation from Structural Relaxation}

Angell (1) has introduced a decoupling index $R_{\tau}, 10^{0}<R_{\tau}<10^{13}$, which describes how well the ion conductivity relaxation process is decoupled from the glassy matrix. The decoupling index is defined as $R_{\tau}\left(T_{g}\right)=\left\langle\tau_{s}\left(T_{g}\right)>1<\tau_{\sigma}\left(T_{g}\right)>\right.$ where $\left\langle\tau_{s}\left(T_{g}\right)>\right.$ and $\left\langle\tau_{\sigma}\left(T_{g}\right)>\right.$ are the average structural relaxation and conductivity relaxation times respectively at the glass transition temperature $T_{g}$. Standard definition of the glass transition temperature fixes the structural relaxation time $\left\langle\tau_{\mathrm{s}}\left(\mathrm{T}_{\mathrm{g}}\right)\right\rangle=200$ seconds. Therefore, the ratio $R_{\tau}$ conveniently describes the extent to which the ion conducting motions in a given glass can be considered decoupled from the viscous motions of the glassy matrix. In some systems (30) the ratio $\left\langle\tau_{\mathrm{S}}(\mathrm{T})\right\rangle /\left\langle\tau_{\sigma}(\mathrm{T})\right\rangle=\mathrm{R}_{\tau}(\mathrm{T})$ has been obtained over a wide range of temperatures at and above $\mathrm{T}_{\mathrm{g}}$. High above $\mathrm{T}_{g}, \mathrm{R}_{\tau}(\mathrm{T})$ is typically of order unity for practically all glass-forming ionic systems. As the liquid is cooled to $T_{g}, R_{\tau}(T)$ in general increases but the pattern of increase as $T$ approaches $T_{g}$ may vary from system to system. Clearly two systems having very different $R_{\tau}$ values at $T_{g}$ necessarily will exhibit different patterns of increase of $R_{\tau}(T)$ with scaled temperature $T_{g} / T$.

Recently Angell (1) proposed that there is a correlation of the nonexponentiality parameter $\beta$ (in the glassy state) that appears in the decay function $\phi(t)=\exp \left[-\left(t / \tau_{\mathrm{s}}^{*}\right)^{\beta}\right]$ of conductivity relaxation $(30,42,43)$ with the decoupling index $R_{\tau}\left(T_{g}\right)$. He found that $\beta$ decreases with increasing $\log R_{\tau}$. Earlier Martin and Angell (62) found a correlation of $\beta$ with $E_{a}^{*}$ in the systems $x L i_{2} \mathrm{O} \cdot(1-x) \mathrm{P}_{2} \mathrm{O}_{5}$. For $0.368<x<0.625, \beta$ increases with $\mathrm{E}_{\mathrm{a}}^{*}$. This correlation was considerably extended by Ngai et al to nearly al alkali and mixed alkali oxide glasses in which $\beta$ and $E_{\mathfrak{a}}^{*}$ have been measured. Moreover, an 
even better correlation of $\beta$ with the "primitive" activation energy $E_{a}=\beta E_{a}^{*}$ was found. A strong correlation of $\beta$ with $E_{a}$ ( and a weaker correlation of $\beta$ with $E_{A}^{*}$ ) is expected from the coupling model for reasons as described by Ngai and Martin (10). Here we shall point out that the newly proposed correlation of $\beta$ with $R_{\tau}\left(T_{g}\right)$ is also expected by the coupling model.

A glass with smaller $R_{\tau}\left(T_{g}\right)$ means the ion-conducting motions in it are more coupled to the viscous motions related to structural relaxation of the matrix. In the glass transition region near $T_{g}$, the apparent activation energy of structural relaxation is very large for $T \geq T_{g}$ where $\left\langle\tau_{S}(T)\right\rangle$ has the VogelFulcher temperature dependence of $\left\langle\tau_{\mathrm{S}}(\mathrm{T})\right\rangle=\mathrm{A} \exp \left[\mathrm{B} / \mathrm{R}\left(\mathrm{T}-\mathrm{T}_{\mathrm{O}}\right)\right]$. For $\mathrm{T}<\mathrm{T}_{\mathrm{g}}$ in the glassy state, the structure has fallen out of equilibrium and $\left\langle\tau_{\mathrm{s}}(\mathrm{T})>\right.$ has an Arrhenius temperature dependence of $<\tau_{\mathrm{s}}(\mathrm{T})$ $>=\mathrm{C} \exp (\mathrm{H} / \mathrm{RT})$, where the activation enthalpy $\mathrm{H}$ of structural relaxation is usually quite large. It is clear that the more coupled the ion-conducting motions are to the viscous structural motions, the closer the activation energy $\mathrm{E}_{\mathrm{a}}^{*}$ of ionic dc conduction in the glass will be to $\mathrm{H}$. In other words, glasses with smaller $R_{\tau}\left(T_{g}\right)$ will have larger $E_{a}^{*}$. From the definition of $R_{\tau}\left(T_{g}\right)$, the expression $\left\langle\tau_{\sigma}\left(T_{g}\right)\right\rangle=9 \times 10^{-}$ $13 / \sigma_{\mathrm{dc}}\left(\mathrm{T}_{\mathrm{g}}\right)$ sec. (Angell, 1986) and $\left\langle\tau_{\mathrm{s}}\left(\mathrm{T}_{\mathrm{g}}\right)>=200 \mathrm{sec}\right.$. we can write down the relation $\mathrm{R}_{\tau}\left(\mathrm{T}_{\mathrm{g}}\right)=2.22 \mathrm{x}$ $10^{14} \sigma\left(\mathrm{T}_{\mathrm{g}}\right)$ where $\sigma\left(\mathrm{T}_{\mathrm{g}}\right)$ has the dimension of ohm-1 $\mathrm{cm}^{-1}$. Thus, the correlation of $R_{\tau}\left(\mathrm{T}_{\mathrm{g}}\right)$ with $\mathrm{E}_{\mathrm{a}}^{*}$ is equivalent to the correlation of $\sigma_{\mathrm{dc}}\left(\mathrm{T}_{\mathrm{g}}\right)$ with $\mathrm{E}_{\mathrm{a}}^{*}$. The latter correlation was pointed out by Hunter and Ingram (63) for $\mathrm{Na}^{+}$-ion conducting glasses.

Additional examples were provided (64) to support the contention that both correlations, $\beta$ with $\mathrm{R}_{\tau}\left(\mathrm{T}_{\mathrm{g}}\right)$ and $\beta$ with $\mathrm{E}_{\mathrm{\lambda}}^{*}$, hold simultaneously. We prefer to examine the correlations in each class of ionic glasses separately first, before we examine the question whether these correlations continue to hold when ionic glasses form all classes with diverse chemical structures and compositions are considered all together. In each case, both correlations of $\beta$ with $\log R_{\tau}\left(T_{g}\right)$ and $\beta$ with $E_{a}^{*}$ are found to be valid. There is a special significance in verifying both correlations simultaneously in ionic glasses with constant ion concentration because the variation of $\beta$ here is not obviously related to change in ion-ion separation. We expect the correlation of a decrease of $\beta$ with increase in $\log R_{\tau}\left(T_{g}\right)$. This is because the more decoupled the ions are from the system matrix, the more important is the many body correlations between the ions have in slowing down the conductivity relaxation. In the coupling model the degree of slowing down is measured by the coupling parameter $n$ which can be identified with 1- $\beta$. Hence from these arguments it follows that an increase in $\log R_{\tau}(\mathrm{Tg})$ will be accompanied by an increase in $n$ or a decrease in $\beta$.

\section{Summary and Discussions}

In this work we brought out some of the latest developments in the dynamics of ionic motions in glassy ionic conductors. Monte Carlo simulations of interacting ions in glasses have provided more insight into the dynamics. We show here that the results of these Monte Carlo simulations are in total agreement with the results and predictions of the coupling model which were published earlier. Two recent, important, experimental developments are brought out for discussion here. One is NMR spinlattice relaxation measurements of alkali oxide glasses which are poor ionic conductors. The other is quasi-elastic neutron scattering experiments performed in fast ionic conductors. In both experiments an activation energy, $E_{a}$, related to ionic motion but significantly smaller than the dc conductivity activation energy, $E_{n}^{*}$, has been directly measured. We show that $E_{a}$ has to be interpreted as the true one-particle activation energy of ion motion before ion-ion interactions are taken into account, while the larger $E_{a}^{*}$ is the apparent activation energy due to the slowing down of one-particle relaxation rate by cooperativity between the ions in glassy ionic conductors. These two activation energies, according to the coupling model, are related through the Kohlrausch exponent $\beta \equiv(1-n)$ of the conductivity relaxation function by the relation $\beta E_{\mathrm{a}}^{*}=\mathrm{E}_{\mathrm{a}}$. NMR spin-lattice relaxation data of the alkali oxide glasses as well as the quasielastic neutron scattering data of two $\mathrm{Ag}$ ion containing, fast glassy conductors have verified this prediction and confirmed the physical interpretations of these two activation energies in the framework of the coupling model. 


\section{Acknowledgement}

This work is supported in part by ONR N0001491WX24019.

\section{References}

/1/ See papers in Proceedings of International Discussion Meeting on Relaxations in Complex Systems, Heraklion, Crete 1990, J. Non-Cryst. Solids 131 - 133 (1991).

12/ Angell, C.A, Chem. Rev. 90 (1990) 523.

13/ Ngai, K.L, Non-Debye Relaxations in Condensed Matter, Ramakrishnan, T.V, and Laksmi, Raj, World Scientific, (1987) 12.

14/ Ngai, K.L, Solid State Ionics 5 (1981) 27.

15/ Ngai, K.L, and Jain, H, Solid State Ionics 18 \& 19 (1986) 362.

16) Ngai K.L, and Rendell, R.W, Handbook of Conducting Polymers, Skotheim, T.A, editor, Dekker, Marcel, New York 2 (1986c) 967.

II Ngai, K.L, Rendell, R.W, and Jain, H, Phys. Rev B 30 (1984) 2133.

18/ Ngai, K.L Rajagopal, A.K, Rendell, R.W, and Teitler, S, IEEE Trans. Electrical Insulations, EI21 (1986) 313; Ngai, K.L, Rendell, R.W, Rajagopal, A.K, and Teitler, S, Annals New York Acad. Sci. 484 (1986) 150. More advances in several fields have been made since 1986, although a review is not available at this time.

19/ Balzer-Jöllenbeck, B, Kanert, O, Jain, H, and Ngai, K.L, Phys. Rev. B 39 (1989) 6071.

/10/ Ngai, K.L, and Martin, S.W, Phys. Rev. 40 (1989) 10550.

/11/ Strom, W, Ngai, K.L, and Kanert, O, J. Non-Cryst. Solids 131 - 133 (1991) 1011; Ngai, K.L, Strom, U, and Kanert, O, Phys. Chem. Glasses (1992) in press.

/12/ Ngai, K.L, and Strom, U, Phys. Rev. B 38 (1988) 10350.

113/ Ngai, K.L, Comments Solid State Phys. 9 (1979) 121; Rajagopal, A.K, Ngai, K.L, and Teitler, S, J. Phys. C (1985).

14/ Ngai, K.L, Rajagopal, A.K, and Teitler, S, J. Chem. Phys. 88 (1988) 5086; Rajagopal, A.K, Ngai, K.L, and Teitler, S, J. Non-Cryst. Solids 131 - 133 (1991) 282

/15/ Ngai, K.L, and Rendell, R.W, J. Non-Cryst. Solids 131 - 133 (1991) 233.

/16/ Peng, S.L, Ngai, K.L, and Tsang, K.Y, to be published.

/17/ Funke, K, and Hoppe, R, Solid State Ionics 40-41 (1990) 200.

/18/ Funke, K, Ber. Bunsenges Phys. Chem. 95 (1991) 955.

/19/ Elliott, S.R; and Owens, A.P, Solid State Ionics 27 (1988) 131.

/20/ Elliott, S.R, Mat. Sci. Eng. B 3 (1989) 69.

/21/ Elliott, S.R, and Owens, A.P, Philos. Mag. B 60 (1989) 777.

122/ Elliott, S.R, and Owens, A.P, Phys. Rev. B 44 (1991) 47. In these references the authors used their diffusion-controlled relaxation (DCR) model to explain the nuclear spin relaxation (NSR) data of the alkali oxide glasses published in References (9), (36) and (55). They tried to understand the ionic concentration dependence of $1 / T_{1}$ by their model (in the version in which the iffusion-independent term $1 / \tau$ is neglected). We point out here that the treatment given by these authors are not consistent with all the facts known from the experimental data. On the one hand their DCR model predicts a superlinear Larmor frequency dependence of $1 / T_{1} \propto \omega_{\mathrm{L}}^{1.5}$, while on the other hand the experimental data of these poor ionic conductors indicate a sublinear Larmor frequency dependence as shown in Eq. (17). Similar problem occurred when the Funke's model is applied directly to explain the spin lattice relaxation data of these poor ionic conductors. Indeed Kanert and coworkers, Reference (36), had pointed out that the observed sublinear frequency dependence at all concentrations $\mathrm{x}$ in $\mathrm{xLi}_{2} \mathrm{O}-(1-\mathrm{x}) \mathrm{GeO}_{2}$ is not in accord with that predicted by Funke's model. These remarks apply also to the model by Elliott and Owen. Any attempt to explain the NSR data of these poor ionic conductors by model based on ionic motions alone will experience the same difficulty. This is the reason why we proposed the TLS mechanism which 
relaxes the nuclear spin and gives rise to the sublinear frequency dependence. At higher temperatures, in addition to the TLS transition, there is also an ion hop and the latter gives rise to the Arrhenius temperature dependence $\exp -\left(\mathrm{E}_{\mathrm{a}} / \mathrm{kT}\right)$ where $\mathrm{E}_{\mathrm{a}}$ is the one-particle activation energy. The activation energy obtained from the NSR data of Kanert et al in the alkali oxide glasses is identified therefore with the true one-particle (primitive) activation energy of ion motion appearing in the coupling model. This is how we made the connection between the NSR data, the explanation by the TLS mechanism, and the coupling model. The exact meaning of this connection was misunderstood by Elliott and Owens and has led them to question the consistency between the TLS mechanism for NRS and the coupling model (see end of page 51 of their paper).

23/ Schirmacher, W, Solid State Ionics 28-30 (1988) 129.

124/ Hunt, A, J. Phys. CM 2 (1990) 9055.

125/ Dyre, J, J. Appl. Phys. 64 (1988) 2456.

126/ Johari, G.P, and Pathmanathan, K, Phys. Chem. Glasses 29 (1988) 219.

/27/ Maass, P, Petersen, J, Bunde, A, Dieterich, W, and Roman, H.E, Phys. Rev. Lett. 66 (1991) 52.

/28/ Bunde, A, and Maass, P, J. Non. Cryst. Solids 131 - 133 (1991) 1022.

129/ Petersen, J, and Dieterich, W, (1991) preprint, to be published in Solid State Ionics.

130/ Howell, F.S, Bose, R.A, Macedo, P.B, and Moynihan, C.T, J. Phys. Chem. 78 (1974) 639.

B1/ Moynihan, C.T, Bressel, R.D, and Angell, C.A, J. Phys. Chem 55 (1971) 4414.

132/ See Moynihan, C.T, in Discussion Section J. Non-Cryst. Solids 131 - 133 (1991) 1117.

133/ Ngai, K.L, in Fast Ion Transport in Solids, Vashista, P, Mundy, J.N, and Shenoy, G.K, editors, (1979) 203.

134/ Balzer-Jöllenbeck, G, Kanert, O, Steinert, J, and Jain, H, Solid State Commun, G5 (1988) 1303.

/35/ Heitjans, P, Faber, W, and Schirmer, A, J. Non-Cryst. Solids 131 - 133 (1991) 1053.

/36/ Kanert, O, Steinert, H, Jain, H, and Ngai, K.L, J. Non-Cryst. Solids 131 - 133 (1991) 1001.

137/ Owens, A.P, Pradel, A, Ribes, M, and Elliott, S.R, J. Non-Cryst. Solids 131 - 133 (1991) 1104; Material Science Society Symposium, Boston Dec. (1991).

138/ Dianoux, A.J, Tachez, M, Mercier, R, and Malugani, J.P, J. Non-Cryst. Solids 131 - 133 (1991) 973.

139/ Ngai, K.L, Rendell, R.W, and Plazek, D.J, J. Chem. Phys. (1990).

140/ Ngai, K.L, "Basic Features of the Glassy State", Colmenero, J, and Allegria, A, editors, World Scientific, Singapore (1990) 265.

141/ Ngai, K.L, and Liu, F.S, Phys. Rev. B 24, (1981) 1049; Rendell, R.W, and Ngai, K.L, J. NonCryst. Solids $131-133$ (1991) 667.

/42/ Macedo, P.B, Moynihan, C.T, and Bose, R, Phys. Chem. Glasses 13 (1972 171.

/43/ Moynihan, C.T, Boesch, L.P, and Laberge, N.L, Phys. Chem. Glasses 14 (1973) 122.

144/ Jain, H, J. Non-Cryst. Solids 131 - 133 (1991) 961.

/45/ Schmidt-Rohr, K, and Spiess, H.W, Phys. Rev. Lett. 66 (1991) 3020.

146/ Ngai, K.L, and Skolnick, J, Macromolecules 24 (1991) 1561.

147/ Pakula, T, J. Non-Cryst. Solids 131 - 133 (1991) 289.

148/ Ngai, K.L, Comments Solid State Phys. 9 (1991) 141.

149/ Martin, S.W, Mat. Chem. Phys. 23 (1989) 225. Spin relaxations rate based on the stretched exponential (KWW) correlation function was worked out for the first time by K.L. Ngai in References (33), (4), (3), (48), and applied to $\mathrm{Na} \beta$-alumina more than a decade ago. At that time in the field of fast ionic conductors the interest was focussed on the alkali $\beta$-aluminas. Extensive NMR data were available for the alkali $\beta$-aluminas but not for fast ionically conducting glasses. It goes without saying that the formalisms as well as the result given in the above references apply verbatim to the fast ionically conducting glasses. In the reference quoted here, S.W. Martin revisited this problem with emphasis on ionic glasses. His results are exactly the same as given by Ngai.

150/ Chung, S.H, Jeffrey, R, Stevens, J.R, and Borgesson, L, Phys. Rev. B 41 (1990) 6154.

/51/ Roos, J, Brinkmann, D, Mali, M, Pradel, A, and Ribes, M, Solid State Ionics 28 - 30 (1988) 710.

152/ Szeftel, J, and Alloul, H, Phys. Rev. Lett. 34 (1975) 657.

153/ Rubinstein M., Resing, H.A, Reinecke, T.L, and Ngai, K.L, Phys. Rev. Lett. 34 (1975) 1444.

/54/ Reinecke, T.L, and Ngai, K.L, Phys. Rev. B 12 (1975) 3476. 
/55/ Ngai, K.L, Mundy, J.N, Jain, H, Kanert, O, and Balzer-Jöllenbeck, Phys. Rev. B 39 (1989) 6169.

156/ Pradel, A, and Ribes, M, J. Non-Cryst. Solids 131 - 133 (1991) 1063.

157/ Burns, A, Chryssikos, G.D, Tombari, E, Cole, R.H, and Risen, W.M, Jr, Phys Chem. Glasses 30 (1989) 264.

/58/ Börgesson, L, , L.M, Martin, S.W, Liu, C, and Angell, C.A, Phys. Lett. A 125 (1987) 330.

159/ Ngai, K.L, and Rendell, R.W, Phys. Rev. B 38 (1988) 9987.

/60/ Torell, L.M, Phys. Rev. B 31 (1985) 4103.

/61/ Liu, C, and Angell, C.A, J. Non-Cryst. Solids 83 (1986) 162.

/62/ Martin, S.W, and Angell, C.A, J. Non-Cryst. Solids 83 (1986) 185.

163/ Hunter, C.C, and Ingram, M.D, Solid State Ionics 14 (1984) 31

/64/ Ngai, K.L, and Mundy, J.N, J. Phys. Chem. Glasses (in press). 